\title{
ON A SIMPLE TYPE OF IRREGULAR SINGULAR POINT*
}

\author{
BY \\ GEORGE D. BIRKHOFF
}

Introduction.

A simple type of irregular singular point is furnished by the linear differential equation of the second order

$$
\frac{d^{2} y}{d x^{2}}+p(x) \frac{d y}{d x}+q(x) y=0
$$

in which the coefficients $p(x)$ and $q(x)$ are analytic at $x=\infty$. The point $x=\infty$ is then an irregular singular point of rank 1 unless $x p(x)$ and $x^{2} q(x)$ remain finite at infinity. If we write $\lim _{x=\infty} p(x)=p_{0}, \lim _{x=\infty} q(x)=q_{0}$, and if the roots $b_{1}, b_{2}$ of the quadratic equation

$$
b^{2}+p_{0} b+q_{0}=0
$$

are distinct, the transformation of variables from $x, y$ to $\bar{x}, \bar{y}$,

$$
y=e^{b_{1} x} \cdot x^{c_{1}} \bar{y}, \quad x=\bar{x}\left(b_{2}-b_{1}\right),
$$

will take (1) into another equation of the same form, in which however

$$
p(x)=-1+{ }_{x}^{p_{1}}+\cdots, \quad q(x)=\frac{q_{2}}{x^{2}}+\frac{q_{3}}{x^{3}}+\cdots,
$$

provided that a proper determination of the constant $c_{1}$ be made.

In the present paper I wish to consider the solutions of (1) in the vicinity of $x=\infty$, but under the restriction that $p(x)$ and $q(x)$ have the form (2). $\dagger$ The method of attack is essentially the same as that which I have employed earlier in the consideration of singular points. $\ddagger$ In this special case the results may be given a more striking form. The last two theorems have no analoguesin my earlier paper and are susceptible of wide generalization.

* Presented to the Society, September 12, 1911.

$\dagger$ This type of equation has been considered in a paper by HorN, J o u r n a l f ü r M a t h e m a tik, vol. 133 (1908), pp. 19-67.

$\ddagger$ These Transactions, vol. 10 (1909), pp. 436-470. 
The treatment will be based on the following auxiliary theorem:* Let $l_{11}(x), l_{12}(x), l_{21}(x), l_{22}(x)$ be functions single-valued and analytic for $|x| \geqq R$ (but not necessarily at $x=\infty$ ) and such that $l_{11}(x) l_{22}(x)-$ $l_{12}(x) l_{21}(x)$ does not vanish for $|x| \geqq R$. Then there exists a set of functions $a_{11}(x), a_{12}(x), a_{21}(x), a_{22}(x)$ analytic at $x=\infty$ and reducing to 1 , $0,0,1$ respectively at $x=\infty$, and a set of entire functions $e_{11}(x), e_{12}(x)$, $e_{21}(x), e_{22}(x)$ for which $e_{11}(x) e_{22}(x)-e_{12}(x) e_{21}(x)$ is nowhere zero in the finite plane, such that

$$
\begin{aligned}
& l_{11}(x)=\left[a_{11}(x) e_{11}(x)+a_{12}(x) e_{21}(x)\right] x^{k_{1}}, \\
& l_{12}(x)=\left[a_{11}(x) e_{12}(x)+a_{12}(x) e_{22}(x)\right] x^{k_{2}}, \\
& l_{21}(x)=\left[a_{21}(x) e_{11}(x)+a_{22}(x) e_{21}(x)\right] x^{k_{1}}, \\
& l_{22}(x)=\left[a_{21}(x) e_{12}(x)+a_{22}(x) e_{22}(x)\right] x^{k_{2}},
\end{aligned}
$$

where $k_{1}$ and $k_{2}$ are integers.

\section{\$1. The Canonical Form of Equation.}

If we set $x(d y / d x)=z$, we may replace the single equation (1) by the linear differential system

$$
\frac{d y}{d x}=\frac{1}{x} z, \quad \frac{d z}{d x}=-x q(x) y+\left(-p(x)+\frac{1}{x}\right) z .
$$

Let us now make a linear transformation of the dependent variables

$$
y=a_{11}(x) Y+a_{12}(x) Z, \quad z=a_{21}(x) Y+a_{22}(x) Z,
$$

where the functions $a_{11}(x), a_{12}(x), a_{21}(x), a_{22}(x)$ are as yet unspecified functions for which $\Delta(x)=a_{11}(x) a_{22}(x)-a_{12}(x) a_{21}(x)$ is not identically zero. The new linear differential system in $Y, Z$ will be

$$
\frac{d Y}{d x}=P_{11}(x) Y+P_{12}(x) Z, \quad \frac{d Z}{d x}=P_{21}(x) Y+P_{22}(x) Z,
$$

where the coefficients of $Y$ and $Z$ have the values

* This theorem is the special case $n=2$ of a theorem which I have proved, $M$ a $\mathrm{t} \mathrm{h} \mathrm{e-}$ mat is che Annalen, vol. 74 (1913), pp. 122-133. This proof, based on the theory of linear integral equations, was first presented by me in 1911 (see B ulletin of the A merican M a thematical Societ y, vol. 18, p. 64) although I was in possession of it a year or two earlier; a proof by an entirely different method was given by me in 1908 (see these Transactions, vol. 10 (1909), p. 438). I have recently noticed that the theorem is a special case of a very important theorem due to Hibsert, Göt inger $\mathrm{Nach}$ richten (1905), pp. 307-330, and Plemew, M on a t shef te für Mathematik und Physik, vol. 19 (1908), pp. 211-222. This relationship becomes obvious if one identifies the functions $l_{i j}(x)$ with the functions $\alpha_{i j}(x)$ in PLEMeLJ's paper, and solves the equations like (3) for $a_{i j}(x)$; for the boundary curve (Randkurve) may be taken the curve $|x|=R$. Furthermore the method used in my second proof is essentially the same as that used by them. 


$$
\begin{aligned}
& P_{11}=\frac{1}{\Delta}\left[a_{22}\left(\frac{1}{x} a_{21}-a_{11}^{\prime}\right)-a_{12}\left(-x q a_{11}+\left(-p+\frac{1}{x}\right) a_{21}-a_{21}^{\prime}\right)\right], \\
& P_{12}=\frac{1}{\Delta}\left[a_{22}\left(\frac{1}{x} a_{22}-a_{12}^{\prime}\right)-a_{12}\left(-x q a_{12}+\left(-p+\frac{1}{x}\right) a_{22}-a_{22}^{\prime}\right)\right], \\
& P_{21}=\frac{1}{\Delta}\left[-a_{21}\left(\frac{1}{x} a_{21}-a_{11}^{\prime}\right)+a_{11}\left(-x q a_{11}+\left(-p+\frac{1}{x}\right) a_{21}-a_{21}^{\prime}\right)\right], \\
& P_{22}=\frac{1}{\Delta}\left[-a_{21}\left(\frac{1}{x} a_{22}-a_{12}^{\prime}\right)+a_{11}\left(-x q a_{12}+\left(-p+\frac{1}{x}\right) a_{22}-a_{22}^{\prime}\right)\right] .
\end{aligned}
$$

The accent here denotes differentiation, and for brevity the letter $x$ is suppressed in the functional notation. If the functions $a_{11}(x), a_{12}(x), a_{21}(x)$, $a_{22}(x)$ are analytic at $x=\infty$ and reduce to $1,0,0,1$ respectively at $x=\infty$, the function $\Delta(x)$ reduces to 1 at $x=\infty$, and a direct computation by means of (7) gives

$$
\begin{aligned}
& P_{11}(x)=\frac{p_{11}^{(2)}}{x^{2}}+\frac{p_{11}^{(3)}}{x^{3}}+\cdots, \\
& P_{12}(x)=\frac{r}{x}+\frac{p_{12}^{(2)}}{x^{2}}+\cdots, \\
& P_{21}(x)=\frac{s}{x}+\frac{p_{21}^{(2)}}{x^{2}}+\cdots, \\
& P_{22}(x)=1+\frac{1-p_{1}}{x}+\frac{p_{22}^{(2)}}{x^{2}}+\cdots
\end{aligned}
$$

Later we shall make use of these expansions in series.

By the aid of the auxiliary theorem above stated we shall choose the transformation (5) so that the equations (6) reduce to a very simple form.

Consider the solutions of (1) in the vicinity of $x=\infty$. According to the fundamental existence theorem for ordinary linear differential equations, every solution of (1) will be analytic for $|x| \geqq R$, if $R$ is chosen so large that $p(x)$ and $q(x)$ are analytic for $|x| \geqq R$; and the general solution will be $c_{1} y_{1}+c_{2} y_{2}$, where $c_{1}, c_{2}$ are arbitrary constants and $y_{1}, y_{2}$ are any pair of linearly independent particular solutions. But $y_{1}$ and $y_{2}$ are not in general single-valued for $|x| \geqq R$; in fact, when $x$ makes a positive circuit of $x=\infty$, $y_{1}$ and $y_{2}$ are only known to be replaced by some pair $\bar{y}_{1}, \bar{y}_{2}$ of linearly independent solutions so that

$$
\bar{y}_{1}=c_{1} y_{1}+c_{2} y_{2}, \quad \bar{y}_{2}=d_{1} y_{1}+d_{2} y_{2}, \quad c_{1} d_{2}-c_{2} d_{1} \neq 0 .
$$

Thus it is evident that $y_{1}, y_{2}$ undergo a linear transformation when $x$ makes a positive circuit of $x=\infty$. According to the well-known facts concerning 
such transformations, it will then be possible so to modify the choice of $y_{1}, y_{2}$ that we have one of the two cases:

$$
\bar{y}_{1}=\rho_{1} y_{1}, \quad \bar{y}_{2}=\rho_{2} y_{2} ; \text {, or } \quad \bar{y}_{1}=\rho_{1} y_{1}, \quad \bar{y}_{2}=\rho_{1} y_{1}+y_{2} .
$$

The second of these alternatives is to be regarded as exceptional $\left(\rho_{1}=\rho_{2}\right)$, and we suppose at present that the first alternative is before us.

If we define $\lambda_{1}$ and $\lambda_{2}$ by means of the equations

$$
\lambda_{1}=\frac{-1}{2 \pi \sqrt{-1}} \log \rho_{1}, \quad \lambda_{2}=\frac{-1}{2 \pi \sqrt{-1}} \log \rho_{2},
$$

thus leaving $\lambda_{1}$ and $\lambda_{2}$ arbitrary up to additive integers, and then define $l_{11}(x), l_{12}(x), l_{21}(x), l_{22}(x)$ by means of the equations

$$
\begin{array}{ll}
y_{1}=x^{\lambda_{1}} l_{11}(x), & y_{2}=x^{\lambda_{2}} l_{12}(x), \\
z_{1}=x \frac{d y_{1}}{d x}=x^{\lambda_{1}} l_{21}(x), & z_{2}=x \frac{d y_{2}}{d x}=x^{\lambda_{2}} l_{22}(x) .
\end{array}
$$

it is clear that the functions $l_{i j}(x)$ are single-valued as well as analytic in the vic nity of $x=\infty$. To see this one has merely to observe that $x^{\lambda_{1}}$ and $x^{\lambda_{2}}$ are respectively multiplied by $\rho_{1}$ and $\rho_{2}$ when $x$ makes a positive circuit of $x=\infty$. Furthermore the expression

$$
l_{11}(x) l_{22}(x)-l_{12}(x) l_{21}(x)=x^{1-\lambda_{1}-\lambda_{2}}\left(y_{1} y_{2}^{\prime}-y_{2} y_{1}^{\prime}\right)=x^{1-\lambda_{1}-\lambda_{2}} e^{-\int p d x}
$$

is not zero for $|x| \geqq R$, since $p(x)$ is analytic. Consequently these functions $l_{11}(x), l_{12}(x), l_{21}(x), l_{22}(x)$ satisfy all the conditions of the auxiliary theorem.

Now let $a_{11}(x), a_{12}(x), a_{21}(x), a_{22}(x), e_{11}(x), e_{12}(x), e_{21}(x), e_{22}(x)$ be the functions and $k_{1}, k_{2}$ the integers fulfilling the fundamental equations (3) of the auxiliary theorem.

The pair of functions $l_{11}(x)$ and $l_{21}(x)$ depend on the initial choice of $\lambda_{1}$. If we multiply the equations (3) whose left-hand members are $l_{11}(x)$ and $l_{21}(x)$ by $x^{-k_{1}}$, these functions are replaced by $x^{-k_{1}} l_{11}(x)$ and $x^{-k_{1}} l_{21}(x)$ respectively. But this pair of functions is the same as the pair $l_{11}(x)$ and $l_{21}(x)$ that would be obtained if $\lambda_{1}$ were replaced by $\lambda_{1}+k_{1}$. A similar remark applies to the pair of functions $l_{12}(x)$ and $l_{22}(x)$. That is, by properly choosing the four functions $l_{11}(x), l_{12}(x), l_{21}(x), l_{22}(x)$ we may take $k_{1}$ and $k_{2}$ equal to zero, and obtain

$$
\begin{aligned}
& l_{11}(x)=a_{11}(x) e_{11}(x)+a_{12}(x) e_{21}(x), \\
& l_{12}(x)=a_{11}(x) e_{12}(x)+a_{12}(x) e_{22}(x), \\
& l_{21}(x)=a_{21}(x) e_{11}(x)+a_{22}(x) e_{21}(x), \\
& l_{22}(x)=a_{21}(x) e_{12}(x)+a_{22}(x) e_{22}(x),
\end{aligned}
$$


where the functions $a_{i j}(x)$ and $e_{i j}(x)$ have the properties stated in the auxiliary theorem.

Let us make this choice of the functions $a_{11}(x), a_{12}(x), a_{21}(x), a_{22}(x)$ in the transformation (5). Multiplying the equations (11) for $l_{11}(x)$, $l_{21}(x)$ and $l_{12}(x), l_{22}(x)$ by $x^{\lambda_{1}}$ and $x^{\lambda_{2}}$ respectively, we obtain

where

$$
\begin{aligned}
& y_{i}=a_{11}(x) Y_{i}+a_{12}(x) Z_{i} \\
& z_{i}=a_{21}(x) Y_{i}+a_{22}(x) Z_{i}
\end{aligned} \quad(i=1,2),
$$

$$
Y_{1}=x^{\lambda_{1}} e_{11}(x), \quad Z_{1}=x^{\lambda_{1}} e_{21}(x), \quad Y_{2}=x^{\lambda_{2}} e_{12}(x), Z_{2}=x^{\lambda_{2}} e_{22}(x) .
$$

Hence, if the transformation (5) be chosen in this way, $\left(Y_{1}, Z_{1}\right)$ and $\left(Y_{2}, Z_{2}\right)$ are a pair of linearly independent solutions of the transformed system (6) of the form (13) where $e_{11}(x), e_{12}(x), e_{21}(x), e_{22}(x)$ are entire functions such that $e_{11}(x) e_{22}(x)-e_{12}(x) e_{21}(x)$ is nowhere zero in the finite plane.

It is now an easy matter to determine explicitly the form of the coefficients $P_{i j}(x)$ in (6). If we substitute the above forms (13) for the known solutions in the first equation (6) we obtain at once

$$
\begin{aligned}
& \frac{d e_{11}(x)}{d x}+\frac{\lambda_{1}}{x} e_{11}(x)=P_{11}(x) e_{11}(x)+P_{12}(x) e_{21}(x), \\
& \frac{d e_{12}(x)}{d x}+\frac{\lambda_{2}}{x} e_{12}(x)=P_{11}(x) e_{12}(x)+P_{12}(x) e_{22}(x) .
\end{aligned}
$$

On account of the fact that the functions $e_{i j}(x)$ are entire, and that $e_{11}(x) e_{22}(x)-e_{12}(x) e_{21}(x)$ is nowhere zero, it follows from these equations that $P_{11}(x)$ and $P_{12}(x)$ are single-valued and analytic everywhere in the finite plane except for a possible pole of the first order at $x=0$; by using the second equation (6) we may likewise show that $P_{21}(x)$ and $P_{22}(x)$ have these same properties. But the functions $P_{i j}(x)$ are analytic at $x=\infty$ according to the formulas (8), which hold inasmuch as $a_{11}(x), a_{12}(x)$, $a_{21}(x), a_{22}(x)$ are analytic at $x=\infty$ and reduce to $1,0,0,1$ respectively at $x=\infty$. Accordingly the functions $P_{i j}(x)$ are linear functions of $1 / x$, and are given by the series (8) which break off at the term in $1 / x$. Therefore the equations (6) have the simple form

$$
x \frac{d Y}{d x}=r Z, \quad x \frac{d Z}{d x}=s Y+\left(x+1-p_{1}\right) Z,
$$

where $r$ and $s$ are certain constants to be studied later. These equations can be integrated by a quadrature in the two cases $r=0$ and $s=0$.

Bearing in mind the nature of the transformation (5) that takes the original equations (4) into (14), and also that $r Z$ and $z$ may be replaced by 
$x(d Y / d x)$ and $x(d y / d x)$ respectively, we are led to summarize our result as follows:

THEOREM I. If $y$ satisfies the equation

$$
\begin{gathered}
\frac{d^{2} y}{d x^{2}}+p(x) \frac{d y}{d x}+q(x) y=0, \\
p(x)=-1+\frac{p_{1}}{x}+\cdots, \quad q(x)=\frac{q_{2}}{x^{2}}+\frac{q_{3}}{x^{3}}+\cdots,
\end{gathered}
$$

then $y$ and $x(d y / d x)$ can be represented in the forms

$$
y=a_{11}(x) Y+a_{12}(x) \frac{x}{r} \frac{d Y}{d x}, \quad x \frac{d y}{d x}=a_{21}(x) Y+a_{22}(x) \frac{x}{r} \frac{d Y}{d x}
$$

where $Y$ is some solution of

$$
\frac{d^{2} Y}{d x^{2}}+\left(-1+\frac{p_{1}}{x}\right) \frac{d Y}{d x}-\frac{r s}{x^{2}} Y=0 \quad(r, s \text { constants })
$$

and $a_{11}(x), a_{12}(x), a_{21}(x), a_{22}(x)$ are analytic at $x=\infty$, reducing to 1 , $0,0,1$ respectively at $x=\infty$. In the case $r=0$ it is necessary to replace

$$
\frac{x}{r} \frac{d Y}{d x} \text { by } \lim _{r=0} \frac{x}{r} \frac{d Y}{d x} \text {. }
$$

The equation (16) is obtained from (14) under the hypothesis $r \neq 0$. When $r$ approaches zero, it is possible to make the solution $(Y, Z)$ of (14) approach any particular solution of that equation for $r=0$, i. e., one may write

$$
Z=\lim _{r \doteq 0} \frac{x}{r} \frac{d Y}{d x}
$$

A slight modification in the proof must be made in case the second alternative (9) is before us. In this event we may define $l_{11}(x), l_{12}(x), l_{21}(x)$, $l_{22}(x)$ so as to satisfy the restrictions of the auxiliary theorem by writing instead of (10)

$$
\begin{aligned}
y_{1} & =x^{\lambda_{1}} l_{11}(x), \quad y_{2}=x^{\lambda_{1}}\left(\frac{l_{11}(x)}{2 \pi \rho_{1} \sqrt{-1}} \log x+l_{12}(x)\right), \\
z_{1}=x \frac{d y_{1}}{d x} & =x^{\lambda_{1}} l_{21}(x), \quad z_{2}=x \frac{d y_{2}}{d x}=x^{\lambda_{1}}\left(\frac{l_{21}(x)}{2 \pi \rho_{1} \sqrt{-1}} \log x+l_{22}(x)\right) .
\end{aligned}
$$

From this point on we may develop the argument as we did before, except for a mere difference of analytical detail.

The equation (16), which comes from the linear system (14) by an elimination of $Z$, may be termed the canonical form of equation (1).

It is clear that a study of the solutions of (1) in the vicinity of $x=\infty$ Trans. Am. Math. Soc. 81 
reduces at once to a study of the solutions of this simple equation (16) which involves only the known constant $p_{1}$ and the product $r s$. The point $x=0$ is a regular singular point of (16) and of (14) with exponents $\lambda_{1}$ and $\lambda_{2}$ by (13). The indicial equation at $x=0$ is

$$
\lambda^{2}+\left(p_{1}-1\right) \lambda-r s=0,
$$

so that we have the relations

$$
\lambda_{1}+\lambda_{2}=1-p_{1}, \quad \lambda_{1} \lambda_{2}=-r s .
$$

One may easily verify that these relations continue to hold even in the second alternative (9) when the multipliers are equal.

\section{§2. The Formal Solutions and the Exponents.}

The equation (1) admits of two formal solutions* of the following types

$s_{1}(x)=\left(1+\frac{s_{1}^{(1)}}{x}+\frac{s_{1}^{(2)}}{x^{2}}+\cdots\right), \quad s_{2}(x)=e^{x} x^{-p_{1}}\left(1+\frac{s_{2}^{(1)}}{x}+\frac{s_{2}^{(2)}}{x^{2}}+\cdots\right)$.

This fact may be verified by a direct substitution which permits one to determine $s_{1}^{(1)}, s_{21}^{(2)}, \cdots$ and $s_{2}^{(1)}, s_{2}^{(2)}, \ldots$ uniquely so as to make $s_{1}(x)$ and $s_{2}(x)$ formally satisfy (1). Moreover the canonical equation (16) is also of this type (1), and in this case an explicit determination of these formal series $S_{1}(x), S_{2}(x)$ gives at once $\dagger$

$$
\begin{aligned}
& S_{1}(x)=\left(1-\frac{\lambda_{1} \lambda_{2}}{1} \cdot \frac{1}{x}+\frac{\lambda_{1}\left(\lambda_{1}+1\right) \lambda_{2}\left(\lambda_{2}+1\right)}{1 \cdot 2} \cdot \frac{1}{x^{2}}+\cdots\right) \\
& S_{2}(x)=e^{x} x^{-p_{1}}\left(1+\frac{\left.\lambda_{1}-1\right)\left(\lambda_{2}-1\right)}{1} \frac{1}{x}\right. \\
&\left.\quad+\frac{\left(\lambda_{1}-1\right)\left(\lambda_{1}-2\right)\left(\lambda_{2}-1\right)\left(\lambda_{2}-2\right)}{1 \cdot 2} \frac{1}{x^{2}}+\cdots\right) .
\end{aligned}
$$

Now, putting aside for the moment the case $r=0$, we see that the formal solutions of the linear differential system (4) are given by

$$
y=s_{1}(x), \quad z=x \frac{d s_{1}(x)}{d x} ; \quad y=s_{2}(x), \quad z=x \frac{d s_{2}(x)}{d x},
$$

* A discussion of such formal solutions is given by HoRN : Gewöhnliche Differentialgleichungen beliebiger Ordnung, pp. 183-188.

t An easy way to obtain the second series is to substitute $y=e^{x} x^{-p_{1}} w$ in (6), and in the resulting transformed equation put $w=\sum_{\nu=0}^{\infty} s_{2}^{(\nu)} x^{-\nu}$. The simplification of the form of the recurrent relation between the successive coefficients in both series depends on (17). 
and that these solutions for (14) are

$$
Y=S_{1}(x), \quad Z=\frac{x}{r} \frac{d S_{1}(x)}{d x} ; \quad Y=S_{2}(x), \quad Z=\frac{x d S_{2}(x)}{r}
$$

It is also clear that each of these sets for $Y, Z$ goes into the corresponding set for $y, z$, by the transformation (5). In fact the formal solutions $Y, Z$ go into formal solutions $y, z$ of the proper form with the same leading terms since the functions $a_{11}(x), a_{12}(x), a_{21}(x), a_{22}(x)$ are power series in negative powers of $x$ with leading coefficients $1,0,0,1$ respectively. It is directly demonstrable that the form and leading terms characterize the formal series for $y, z$ completely, and hence the correspondence must be as stated.

Therefore if we replace $y$ by $s_{i}(x)$ in (15) and $Y$ by $S_{i}(x)$ respectively for $i=1,2$ the resulting equations are formally correct. These relations permit us to determine power series for $a_{11}(x), a_{12}(x), r, a_{21}(x) / r$, $a_{22}(x) / r$ by means of the known series for $s_{i}(x)$ to be obtained from (1), and the series $S_{i}(x)$ given in (18).

These latter series depends on $\lambda_{1}$ and $\lambda_{2}$ alone, so that we are in a position to actually obtain the functions $a_{11}(x), a_{12}(x) / r, a_{21}(x), a_{22}(x) / r$ in (15) as soon as $\lambda_{1}$ and $\lambda_{2}$ are known. That is, once a set of exponents $\lambda_{1}, \lambda_{2}$ are obtained it will be possible to determine the transformation (5) explicitly. We observe that, since $a_{22}(x)$ reduces to 1 at $x=\infty$, the power series for $a_{22}(x) / r$ begins with the term $1 / r$, which permits us to determine $r$; and that when $r$ is found, $s$ may be determined by means of the relation $r s=-\lambda_{1} \lambda_{2}$.

Likewise even if $r$ is zero the equations (14) again admit of similar formal solutions, and by substituting in both sides of (5) corresponding formal solutions of (4) and (14) one may determine $a_{11}(x), a_{12}(x), a_{21}(x), a_{22}(x)$, and $s$.

It is essential therefore to determine the possible values of $\lambda_{1}$ and $\lambda_{2}$, and to know in what cases $r$ may be zero. Concerning these exponents we know at present that

(19) $\quad \lambda_{1}=-\frac{1}{2 \pi v^{\prime}-1} \log \rho_{1}, \quad \lambda_{2}=-\frac{1}{2 \pi v^{\prime}-1} \log \rho_{2}, \quad \lambda_{1}+\lambda_{2}=1-p_{1}$.

Any pair of exponents $\lambda_{1}$ and $\lambda_{2}$ may be replaced by $\lambda_{1}+m$ and $\lambda_{2}-m$ respectively where $m$ is any integer, without destroying the relations (19). The multipliers $\rho_{1}, \rho_{2}$ may be computed in various ways and we shall suppose these to be given quantities.

In order to completely consider all possibilities we need to make a subdivision into two cases, the first of which is the following: If neither of the multipliers $\rho_{1}, \rho_{2}$ reduce to 1 (Case $A$ ) the exponents $\lambda_{1}, \lambda_{2}$ are unrestricted save 
as indicated by (19) and neither $r$ nor $s$ is zero. In this case both formal solutions of (1) diverge.

To prove this we note that there is a transformation of the form (5)

$$
y=b_{11}(x) \bar{Y}+b_{12}(x) \bar{Z}, \quad . Z=b_{21}(c) \bar{Y}+b_{22}(x) \bar{Z},
$$

where $b_{11}(x), b_{12}(x), b_{21}(x), b_{22}(x)$ are analytic at $x=\infty$ and reduce to $1,0,0,1$ respectively at $x=\infty$, that takes the equations (14) into like equations in which the exponents $\lambda_{1}, \lambda_{2}$ are changed to $\lambda_{1}-1, \lambda_{2}+1$ respectively. In fact if we write

$$
\begin{aligned}
& Y=\left(1+\frac{\lambda_{1}-\lambda_{2}-1}{x}\right) \bar{Y}+\frac{\lambda_{1}}{s}\left(\frac{\lambda_{1}-\lambda_{2}-1}{x}\right) \bar{Z}, \\
& Z=\frac{-s}{\lambda_{1}}\left(\frac{\lambda_{1}-\lambda_{2}-1}{x}\right) \bar{Y}+\left(1-\frac{\lambda_{1}-\lambda_{2}-1}{x}\right) \bar{Z},
\end{aligned}
$$

these equations (14) go into

$$
x \frac{d \bar{Y}}{d x}=\frac{\lambda_{1}\left(1-\lambda_{1}\right)}{s} \bar{Z}, \quad x \frac{\bar{d} Z}{d x}=\frac{s\left(1+\lambda_{2}\right)}{\lambda_{1}} \bar{Y}+\left(x+1-p_{1}\right) \bar{Z}
$$

in which $-r s=\lambda_{1} \lambda_{2}$ is replaced by $-\overline{r s}=\left(\lambda_{1}-1\right)\left(\lambda_{2}+1\right)$. It is to be observed that $r$ and $s$ are not zero, for that implies that either $\lambda_{1}$ or $\lambda_{2}$ is zero by (17) so that one of the multipliers is 1 .

By compounding the transformation (5) with the transformation (21) we obtain another transformation of the same form as (5) taking (4) directly to (21). Hence we have increased one of the exponents by unity and decreased the other by unity. By a succession of such steps we can obtain an equation (i4) in which the exponents $\lambda_{1}, \lambda_{2}$ are replaced by $\lambda_{1}+m, \lambda_{2}-m$ where $m$ is any integer. This proves our first statement above.

Now the formal series (18) diverge unless they terminate, and they cannot terminate since neither $\lambda_{1}$ nor $\lambda_{2}$ is an integer, by hypothesis. But it is obvious that corresponding series $s$ and $S$ for (1) and (16) either converge or diverge together by (5). It follows that the two formal solutions of (1) diverge in this case.

The second case is the following: If one of the multipliers, as $\rho_{1}$, reduces to 1 (Case $B$ ) it is possible to take $\lambda_{1}=0$ and $\lambda_{2}=1-p_{1}$. At least one of the formal solutions $s_{1}(x), s_{2}(x)$ of $(1)$ converges. If $s_{1}(x)$ diverges, then $r$ but not $s$ reduces to zero; if $s_{2}(x)$ diverges, $s$ but not $r$ reduces to zero; if both $s_{1}(x)$ and $s_{2}(x)$ converge, we may take $r=s=0$.

In this case one of the exponents is zero at the outset, or by a succession of transformations (20) it will be possible to reduce an integral exponent corresponding to the multiplier 1 to the value zero so that $\lambda_{1}=0$; we shall have then $\lambda_{2}=1-p_{1}$, by (17). 
Also since we have $r s=-\lambda_{1} \lambda_{2}=0$, either $r$ or $s$ is zero.

If $r=0$, the system (14) becomes

$$
x \frac{d Y}{d x}=0, \quad x \frac{d Z}{d x}=s Y+\left(x+1-p_{1}\right) Z .
$$

The two formal solutions of this system are clearly

$$
\begin{array}{ll}
Y=1, & Z=-\frac{s}{x}\left(1+\frac{p_{1}-2}{x}+\frac{\left(p_{1}-2\right)\left(p_{1}-3\right)}{x^{2}}+\cdots\right), \\
Y=0, & Z=e^{x} x^{1-p_{1}},
\end{array}
$$

the second of which is terminating and so convergent. If $s=0$ the system (14) has the two formal solutions

$$
\begin{aligned}
& Y=1, \quad Z=0, \\
& Y=r e^{x} x^{-p_{1}}\left[1+\frac{p_{1}}{x}+\frac{p_{1}\left(p_{1}+1\right)}{x^{2}}+\cdots\right], \quad Z=e^{x} x^{1-p_{1}},
\end{aligned}
$$

by (18), and here the first series is terminating and convergent. In either case one of the formal solutions of (14), and hence the corresponding formal solution of (1) converges. If $s_{1}(x)$ diverges, so will the corresponding solution of (16) and we must have the first case $r=0$. Since $s$ appears as a factor in the first series (23) we infer $s \neq 0$ in this case. Likewise if $s_{2}(x)$ diverges we infer $r \neq 0, s=0$.

It only remains therefore to show that in the case when both $s_{1}(x)$ and $s_{2}(x)$ converge we may take $r=s=0$. We have already seen that either $r$ or $s$ is zero. Suppose we have $r=0, s \neq 0$. An inspection of the first formal solution (23) shows that $p_{1}$ must have one of the values $2,3, \ldots$ in order that the series for $Z$ may terminate and converge. The first solution may be written $Y=1, Z=-s P(x) / x$, where $P(x)$ is a polynomial in $1 / x$. If we make the further transformation

$$
Y=\bar{Y}, \quad Z=-\frac{s}{x} P(x) \bar{Y}+\bar{Z},
$$

of the form (20) where $b_{11}(x), b_{12}(x), b_{21}(x), b_{22}(x)$ are analytic at $x=0$ and reduce to $1,0,0,1$ respectively at $x=\infty$, the resulting system in $\bar{Y}, \bar{Z}$ is

$$
x \frac{d \bar{Y}}{d x}=0, \quad x \frac{d \bar{Z}}{d x}=\left(x+1-p_{1}\right) \bar{Z}
$$

of the form (14) with $r=s=0$. But the transformation (5) and the above transformation are together equivalent to a single transformation of the form (5), so that we may take $r=s=0$. Likewise we may dispose of the case $r \neq 0, s=0$ by a consideration of the second of the solutions (24). 


\section{§3. Explicit Solution of the Canonical Form of Equation.}

In order to study completely the solutions of (1) in the vicinity of $x=\infty$ it is necessary to solve the canonical form (16) of equation equivalent to the linear system (14). If either multiplier $\rho_{1}, \rho_{2}$ is 1 , we have essentially Case $B$ and we may reduce the equation (14) to a form in which $r=0$ or $s=0$. In either case the reduced system (14) is integrable by a quadrature, and we have

$$
\begin{array}{ll}
Y=c, \quad Z=e^{x} x^{1-p_{1}}\left[d+c s \int e^{-x} x^{p_{1}-2} d x\right] & \text { if } r=0, \\
Y=d+c r \int e^{x} x^{-p_{1}} d x, \quad Z=c e^{x} x^{1-p_{1}} & \text { if } s=0 .
\end{array}
$$

Therefore the canonical equations (14) can always be solved by a single quadrature if either $\lambda_{1}$ or $\lambda_{2}$ is an integer.

However when neither $\lambda_{1}$ nor $\lambda_{2}$ is an integer it is possible to effect the solution of (16) by means of the Laplace transformation. To simplify the form of this transformation let us write $Y=x^{\lambda_{1}} \bar{Y}$ in (12) and obtain the equation*

$$
x \frac{d^{2} \bar{Y}}{d x^{2}}+\left(1+\lambda_{1}-\lambda_{2}-x\right) \frac{d \bar{Y}}{d x}-\lambda_{1} \bar{Y}=0 .
$$

This equation is of the simple type to which Laplace first applied his transformation, and using his method we find the formulas

$$
Y_{i}=x^{\lambda_{1}} \int_{l_{i}} e^{x_{z}} z^{\lambda_{1}-1}(1-z)^{-\lambda_{2}} d z \quad \quad(i=1,2),
$$

and likewise by interchanging $\lambda_{1}$ and $\lambda_{2}$, we find

$$
Y_{i}=x^{\lambda_{2}} \int_{l_{i}} e^{x_{2}} z^{\lambda_{8}-1}(1-z)^{-\lambda_{1}} d z \quad(i=1,2) .
$$

Here the contours $l_{1}$ and $l_{2}$ are loop-circuits to the points $z=0$ and $z=1$ respectively taken from $z=\infty$ along a ray to these points, making a circuit in a negative sense and returning to $\infty$ along the ray. The ray must be so chosen that the integrals converge.

Under the conditions that neither $\lambda_{1}$ nor $\lambda_{2}$ is an integer the two solutions of either set are linearly independent.

* Horn has considered the application of the Laplace transformation to the equation

$$
\left(a_{0}+b_{0} x\right) \frac{d^{2} y}{d x}+\left(a_{1}+b_{1} x\right) \frac{d y}{a x}+\left(a_{2}+b_{2} x\right) y=0,
$$

which he reduces first to a normal form somewhat different from our equation (26): $\mathrm{M}$ a t $\mathrm{h}$ emat is che An a le n, vol. 49 (1907), pp. 453-492. The reader who desires to see how the integral given by the Laplace transformation may be made to yield complete information about the solution $Y$ is referred to this interesting paper. 
It is now possible to give more explicit form to the relation (15) of Theorem I which we do in the following:

TheOREM I'. Two linearly independent solutions of an equation

$$
\begin{gathered}
\frac{d^{2} y}{d x^{2}}+p(x) \frac{d y}{d x}+q(x) y=0, \\
p(x)=-1+\frac{p_{1}}{x}+\cdots, \quad q(x)=\frac{q_{2}}{x^{2}}+\frac{q_{3}}{x^{3}}+\cdots
\end{gathered}
$$

may be represented in the form

$$
\begin{array}{r}
y_{i}=x^{\lambda_{1}}\left[a_{i}(x) \int_{l_{i}} e^{x z} z^{\lambda_{1}-1}(1-z)^{-\lambda_{2}} d z+b_{i}(x) \int_{l_{i}} e^{x_{z}} z^{\lambda_{1}}(1-z)^{-\lambda_{2}} d z\right] \\
(i=1,2),
\end{array}
$$

where $a_{i}(x)$ and $b_{i}(x)$ are analytic at $x=\infty$ and reduce to 1 and 0 respectively at $x=\infty$, and $\lambda_{1}, \lambda_{2}$ are any determinations of $\lambda_{1}, \lambda_{2}$ satisfying the equations (18). This representation breaks down only when one of the multipliers $\rho_{1}, \rho_{2}$ reduces to 1 , and then it may be replaced by one of the two following:

$$
\begin{array}{ll}
y_{1}=a\left(x_{1}\right)+b(x) e^{x} x^{1-p_{1}} \int e^{-x} x^{p_{1}-2} d x, & y_{2}=b(x) e^{x} x^{1-p_{1}}, \\
y_{1}=a(x) \int e^{x} x^{-p_{1}} d x+b(x) e^{x} x^{1-p_{1}}, & y_{2}=a(x) .
\end{array}
$$

To prove these relations it is only necessary to write in Theorem I $a_{11}(x)=a(x), a_{12}(x) / r=b(x)$ and to use for $Y$ and $Z$ the forms deduced from (28) and (25).

\$4. Characterization of the Laurent Series for the Solutions.

Let us now restrict ourselves to the non-exceptional case when both of the multipliers $\rho_{1}, \rho_{2}$ are distinct from each other and from 1 . In this case the equation (1) has a pair of solutions $y_{1}(x), y_{2}(x)$ of the forms

$$
y_{1}(x)=x^{\lambda_{1}} l_{11}(x), \quad y_{2}(x)=x^{\lambda_{2}} l_{12}(x),
$$

where $l_{11}(x)$ and $l_{12}(x)$ may be expressed as Laurent series

$$
l_{11}(x)=\sum_{\nu=-\infty}^{+\infty} l_{1}^{(\nu)} x^{\nu}, \quad l_{12}(x)=\sum_{\nu=-\infty}^{+\infty} l_{2}^{(\nu)} x^{\nu},
$$

since these functions are single-valued and analytic for $|x| \geqq R$.

It is an interesting matter to determine the precise character of the coefficients $l_{i}^{(\nu)}$ in these series. To do so we make use of the relations obtained from the transformation (5) taking the linear system (4) to the canonical system (14). The first equation of transformation may be written 


$$
y=a(x) Y+x b(x) \frac{d Y}{d x},
$$

where $Y$ satisfies (14) and $a(x)$ and $b(x)$ are analytic at $x=\infty$ and reduce to 1 and 0 respectively at $x=\infty$. Let us express $a(x)$ and $b(x)$ in the form of power series in negative powers of $x$

$$
a(x)=1+\sum_{\nu=1}^{\infty} a_{\nu} x^{-\nu}, \quad b(x)=\sum_{\nu=1}^{\infty} b_{\nu} x^{-\nu} .
$$

It is clear that the special solutions $Y_{1}$ and $Y_{2}$ of (16) which correspond to the exponents $\lambda_{1}$ and $\lambda_{2}$ go over into the solutions $y_{1}$ and $y_{2}$ respectively, so that we may write

$$
y_{i}=a(x) Y_{i}+x b(x) \frac{d Y_{i}}{d x} \quad(i=1,2) .
$$

But the functions $x^{-\lambda_{1}} Y_{1}, x^{-\lambda_{2}} Y_{2}$ admit of expansion in Laurent series in $x$ convergent for all finite values of $x$, since (16) has no finite singular points except the regular point $x=0$ with exponents $\lambda_{1}, \lambda_{2}$.

If we write $Y=x^{\lambda_{i}} \bar{Y}$ and substitute

in (26) we find that

$$
\bar{Y}_{i}=\sum_{\nu=0}^{\infty} L_{i}^{(\nu)} x^{\nu} \quad(i=1,2),
$$

$$
\begin{aligned}
& Y_{1}=x_{1}^{\lambda_{1}}\left(1+\frac{\lambda_{1}}{1 \cdot\left(\lambda_{1}-\lambda_{2}+1\right)} x+\frac{\lambda_{1} \cdot\left(\lambda_{1}+1\right)}{1 \cdot 2 \cdot\left(\lambda_{1}-\lambda_{2}+1\right) \cdot\left(\lambda_{1}-\lambda_{2}+2\right)} x^{2}+\cdots\right), \\
& Y_{2}=x^{\lambda_{2}}\left(1+\frac{\lambda_{2}}{1 \cdot\left(\lambda_{2}-\lambda_{1}+1\right)}+\frac{\lambda_{2} \cdot\left(\lambda_{2}+1\right)}{1 \cdot 2 \cdot\left(\lambda_{2}-\lambda_{1}+1\right) \cdot\left(\lambda_{2}-\lambda_{1}+2\right)} x^{2}+\cdots\right) .
\end{aligned}
$$

Putting in the explicit series for $y_{i}, a(x), b(x), Y_{i}$ in (29) and comparing coefficients we obtain the following:

THEOREM II. If the multipliers $\rho_{1}, \rho_{2}$ are distinct from each other and from 1 , and if $\lambda_{1}, \lambda_{2}$ are any pair of exponents satisfying the conditions (19), the coefficients $l_{i}^{(\nu)}$ in the Laurent series

$$
y_{1}=x^{\lambda_{1}} \sum_{\nu=-\infty}^{+\infty} l_{1}^{(\nu)} x^{\nu}, \quad y_{2}=x^{\lambda_{2}} \sum_{\nu=-\infty}^{+\infty} l_{2}^{(\nu)} x^{\nu},
$$

in which two particular linearly independent solutions $y_{1}, y_{2}$ of (1) may be expanded, have the form

$l_{i}^{(\nu)}=L_{i}^{(\nu)}+\left[a_{1}+b_{1}\left(\lambda_{i}+\nu+1\right)\right] L_{i}^{(\nu+1)}+\left[a_{2}+b_{2}\left(\lambda_{i}+\nu+2\right)\right] L_{i}^{(\nu+2)}+\cdots$, where 


$$
\begin{aligned}
& L_{1}^{(\nu)}=\frac{\lambda_{1} \cdot\left(\lambda_{1}+1\right) \cdots\left(\lambda_{1}+\nu-1\right)}{1 \cdot 2 \cdots \nu \cdot\left(\lambda_{1}-\lambda_{2}+1\right) \cdot\left(\lambda_{1}-\lambda_{2}+2\right) \cdots\left(\lambda_{1}-\lambda_{2}+\nu\right)} \\
& L_{2}^{(\nu)}=\frac{\lambda_{2} \cdot\left(\lambda_{2}+1\right) \cdots\left(\lambda_{2}+\nu-1\right)}{1 \cdot 2 \cdots \nu \cdot\left(\lambda_{2}-\lambda_{1}+1\right) \cdot\left(\lambda_{2}-\lambda_{1}+2\right) \cdots\left(\lambda_{2}-\lambda_{\nu}+\nu\right)}
\end{aligned}
$$

and $a_{\nu}, b_{\nu}$ are a set of numbers such that $\sqrt[v]{a_{\nu}}$ and $\sqrt[v]{b_{\nu}}$ are limited in absolute value for $\nu=1,2, \cdots$.

The condition on $a_{\nu}$ and $b_{\nu}$ stated in this theorem is equivalent to saying that the series for $a(x)$ and $b(x)$ converge for $|x|$ sufficiently large, according to a well-known test due to Cauchy. The form for $l_{i}^{(v)}$ given in the theorem is sufficient to describe these coefficients completely.

It is not difficult to show that a similar characterization may be made in the special cases not coming under the hypothesis of the theorem.

\section{§5. Functional Relations.}

The theorems which have been developed in the preceding paragraphs are based on a preliminary normalizing transformation of the dependent variable in (1). This disposes of all but two essentially arbitrary constants. The transformation of independent variable $x=\bar{x}+\psi(\bar{x})$, where $\psi(\bar{x})$ is analytic at $\bar{x}=\infty$, leaves (1) unaltered in form but leads to no additional normalization. This fact is expressed in the following:

THEOREM III. If the multipliers $\rho_{1}, \rho_{2}$ are distinct from each other and from 1 , and if the function $\varphi(x)$ has the form $x+\psi(x)$ where $\psi(x)$ is analytic at $x=\infty$, then we have for every solution $Y(x)$ of (16)

$$
Y(\varphi(x))=a(x) Y(x)+b(x) \frac{d Y(x)}{d x}
$$

where $a(x)$ and $b(x)$ are analytic at $x=\infty$.

The proof is immediate. Such a transformation applied to (16) takes that equation into an equation of the form (1) for which $\rho_{1}, \rho_{2}$ are the same as for (16) and whose formal solutions clearly diverge (Case $A$ ) with the corresponding formal solutions of (16), inasmuch as the new formal solutions result from the original formal solutions by this sume transformation $x=\varphi(\bar{x})$. Consequently according to the results of $\S 2$ it is possible to take the canonical equation associated with this transformed equation as in Theorem I to be the same as the equation (16) in $Y$ itself.

If now we replace $y, a_{11}(x)$, and $x a_{12}(x) / r$ by $Y(\varphi(x)), a(x)$, and $b(x)$ respectively in (15) we obtain the equation (33) of the above theorem, where $a(x)$ reduces to 1 at $x=\infty$. Moreover the $Y$ on both sides of equation (33) refers always to one and the same solution, except for a constant 
multiplier; this is a consequence of the fact that $Y(\varphi(x))$ and $Y(x)$ undergo similar transformations when $x$ makes a positive circuit of $x=\infty$. Such a constant multiplier may be absorbed nto $a(x)$ and $b(x)$ so that the modified equation (33) has the desired form.

It is manifest that simple analogues of Theorems II and III exist in the exceptional case when the multipliers become equal or reduce to unity 